\title{
AN IMPROVED AUDIO-FREQUENCY GENERATOR
}

\author{
By E. G. Lapham
}

\section{ABSTRACT}

This paper describes in detail the construction of an audio-frequency generator for use in making radio-frequency measurements. The variable audio-frequency output is the beat note between two sources of radio-frequency; the one a piezo oscillator, and the other a variable oscillator. The output is continuously variable from 50 to 1,500 cycles per second. The entire unit is assembled very compactly and the essential parts are mounted in a temperature-controlled compartment. The calibration curve is practically linear over a range of 50 cycles per second, and repeated calibrations indicate that it is constant to better than 0.1 cycle per second over the entire range.

An audio-frequency oscillator is an essential part of radio-frequency measuring equipment. The last step in such a measurement is the determination of the frequency of the audio beat note between the reference standard and the unknown frequency. If the frequency of the beat note is between 0 and 200 cycles per second, an audio-frequency bridge ${ }^{1}$ provides a very accurate means of making the measurement. However, if the frequency is greater than 200 cycles per second, the most convenient method is to match the note with a known audio-frequency. A variable audio-frequency generator is ordinarily used for this purpose.

The beat-frequency oscillator ${ }^{2}$. provides a very convenient audiofrequency source. The audio-frequency note is the beat between two radio-frequency oscillators; a piezo oscillator and a variable oscillator. The beat note is received by a detector and amplified to the desired energy level by means of an audio-frequency amplifier.

A very satisfactory audio-frequency generator of this type has been constructed for use in measuring the standard frequency transmissions from the National Bureau of Standards radio station, WWV. The complete unit can be seen in Figure 1. It is a very compact unit, mounted in an aluminum case, $18^{3 / 4}$ inches long, $12 \%$ inches high, and $123 / 4$ inches deep. This case is rigidly constructed of three-sixteenthsinch sheet alıminum with all joints fitted neatly to provide an effective radio-frequency shield. In the upper left-hand corner of the front panel is the filament voltmeter and rheostat. At the right of the rheostat is a light which indicates the operation of the heater. The meter in the upper center indicates the current in the piezo oscillator plate circuit, and the one in the upper right, the plate voltage applied to the oscillator tubes. A small toggle switch is seen on either side of the plate milliammeter. The one on the left is connected in the

1 N. P. Case, A Precise and Rapid Method of Measuring Frequencies from 5 to 200 Cycles per Second, B. S. Jour. Research, vol 5 (RP. 195); A ugust, 1930.

A. Hund, Piezo-electric Generator for Audio-Frequencies, B. S. Jour. Research, vol. 2 (RP. 40); February, 1929 
plate circuit of the piezo oscillator, and the one on the right in the plate circuit of the variable oscillator. The outlets directly below the switches provide the corresponding radio-frequency outputs, by means of which either oscillator can be measured separately against a radiofrequency standard, or the piezo oscillator can be used as a secondary standard. A switch, directly below the plate milliammeter, disconnects the filament battery. The audio-frequency output terminals and volume control are located in the lower right-hand corner of the front panel. In the extreme left a door is provided which gives access to a compartment containing a relay, rectifier, and resistors in the heater circuits. The battery cable and the 110-volt a. c. line are connected by means of suitable outlets at the left end of the box.

By unsoldering seven leads that are connected to the circuits in the temperature-controlled compartment, and removing as many screws, the front panel may be removed. The heater control equipment can be seen in Figure 2 at the left. On the right is a condenser dial with a vernier, which allows a slow, smooth adjustment of the frequency of the variable oscillator.

Figure 3 shows the arrangement of tubes and circuits as seen from the rear of the front panel. The two tubes on the right are the oscillator and coupling amplifier in the piezo oscillator circuits. The three tubes in the center are, from right to left, detector, first and second audio-frequency amplifiers. The two tubes on the left are the oscillator and coupling amplifier in the variable oscillator circuits.

Replacing the front panel and removing the top gives a view, as seen in Figure 4, of the interior of the temperature-controlled cabinet. This compartment is $18 \frac{1}{4}$ inches long, 12 inches high, and 7/3/4 inches deep, outside dimensions. The side and end walls, as shown in the drawing (fig. 5), consist of a three-eighths inch layer of hardwood, one-half inch layer of hair-felt, and an inner wall of one-half inch balsa wood. The top and bottom of the cabinet consist of an outer wall of three-eighths inch hardwood and a double layer of one-half inch balsa wood.

The quartz plate and mounting can be seen in the aluminum attenuating box at the left end of the temperature-controlled cabinet. Directly in front, mounted on the balsa wood, is the coil in the piezo oscillator plate circuit. An attenuating cover is placed over this coil to reduce the temperature changes which take place as the heaters operate. The aluminum attenuating box on the right contains the condensers and coils of the variable radio-frequency oscillator. The flexible connection on the condenser shaft is in view between the front of the aluminum box and the balsa wood wall. One of the heaters, which maintain the box at a constant temperature, can be seen at each end of the cabinet. Similar heaters, extending the full length of the cabinet, are placed above and below the attenuating boxes. The thermostat is mounted between the boxes in an aluminum well directly attached to the end of the box containing the quartz plate.

The temperature-controlled cabinet is maintained at $40.5^{\circ} \mathrm{C}$. by means of four electric heaters. The heaters are made of No. 28 nichrome wire which is wound into a long helical spring and then stretched over the forms. The wire is insulated from the metal supports by one-eighth inch porcelain tubing. A sensitive polarized relay is used to operate the heaters. The relay works positively on 2 milliamperes d. c., which is obtained from the a. c. line by means of a copper-oxide 
B. S. Journal of Research, RP367

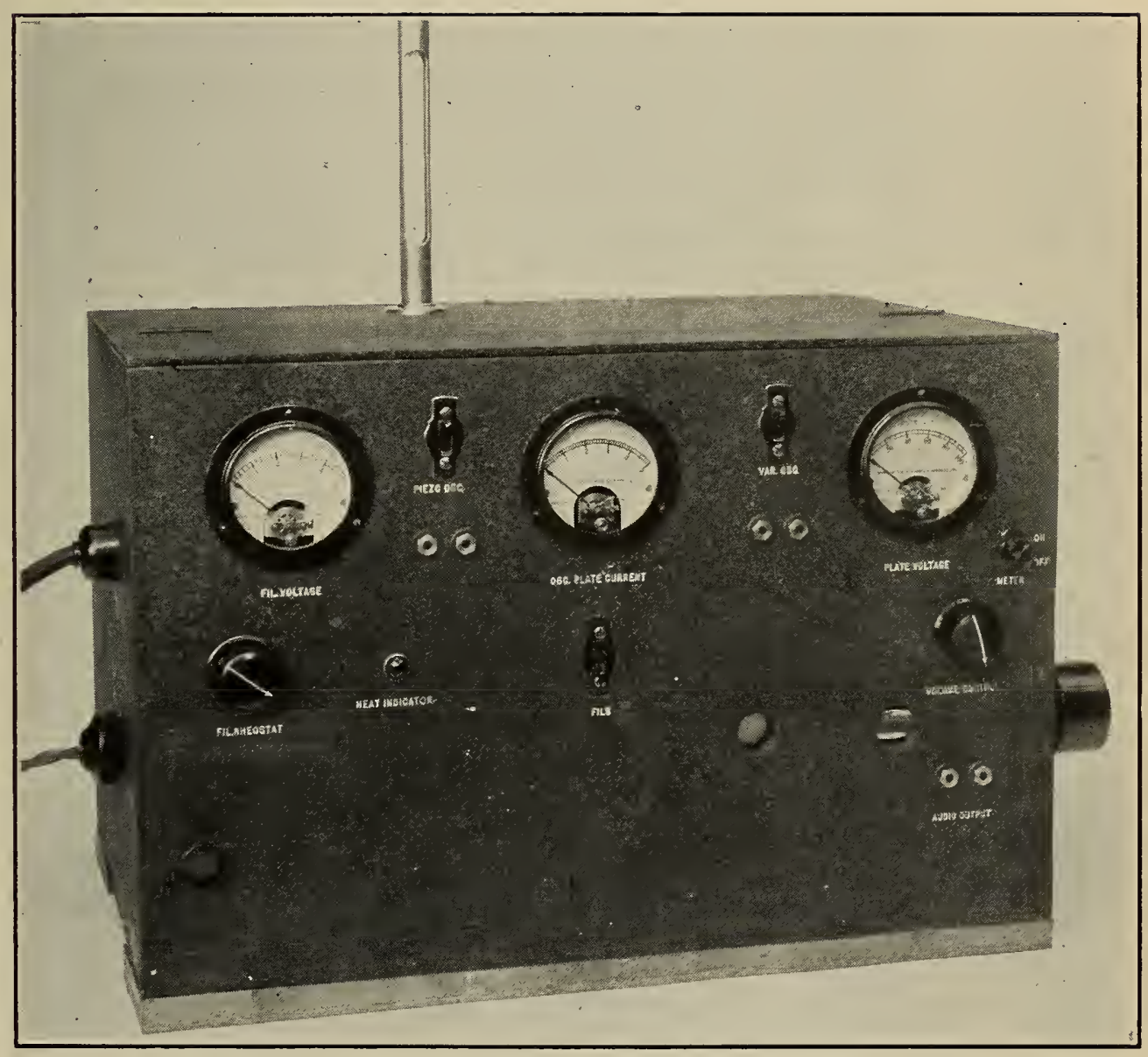

Figure 1.-Front view of audio-frequency generator 
B. S. Journal of Research. RP367

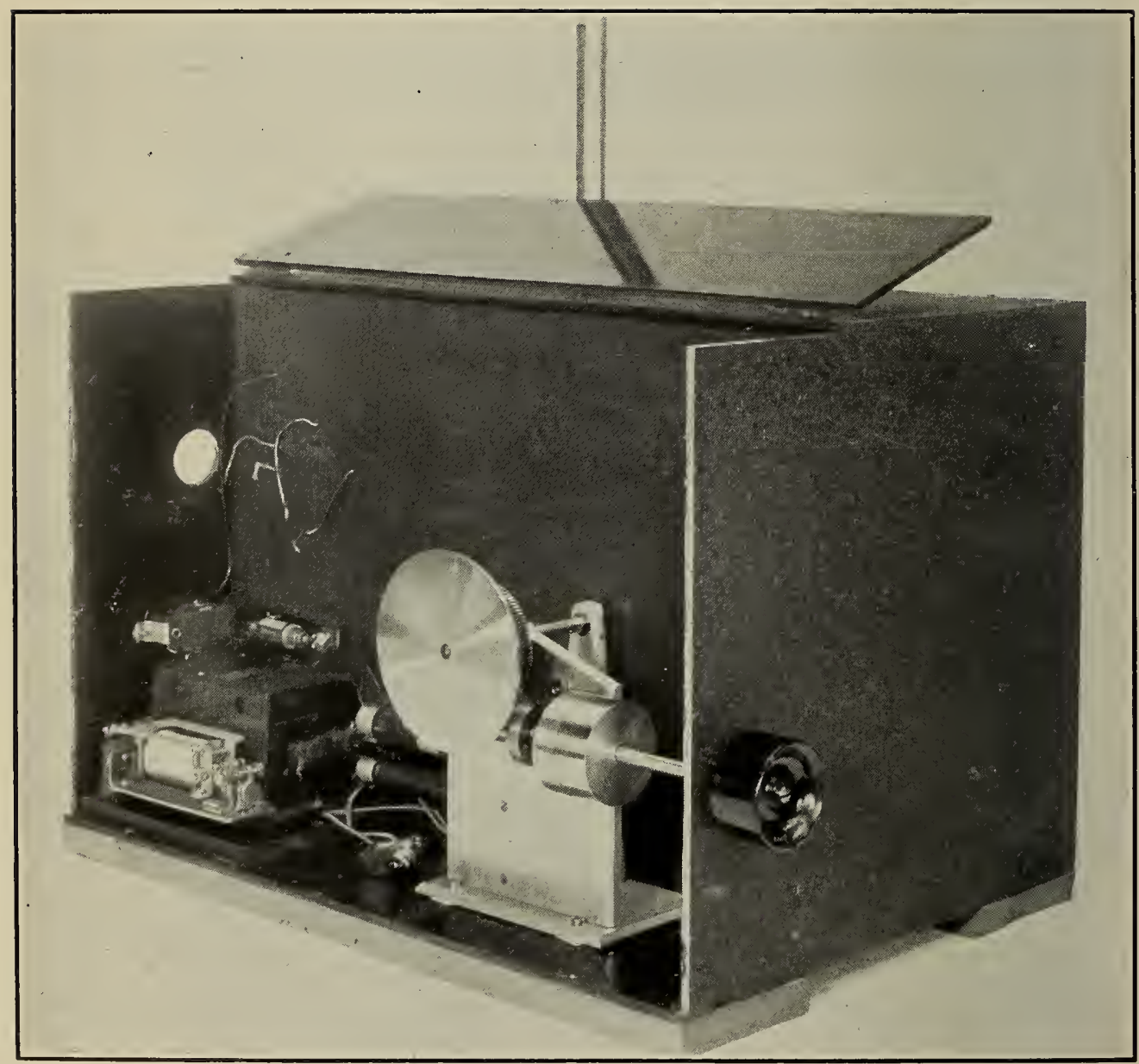

FIGURE 2.-View of audio-frequency generator with front panel removed 


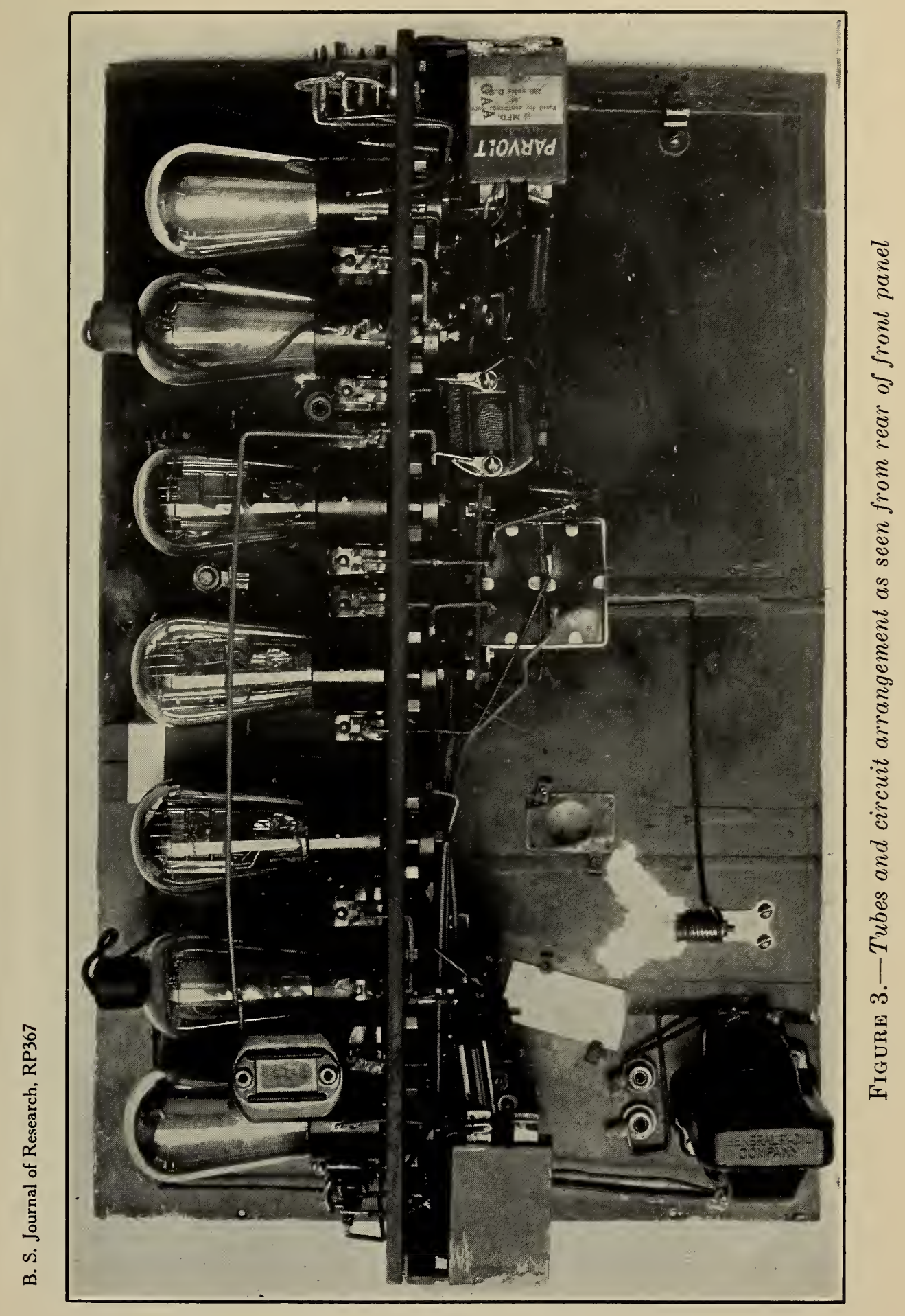




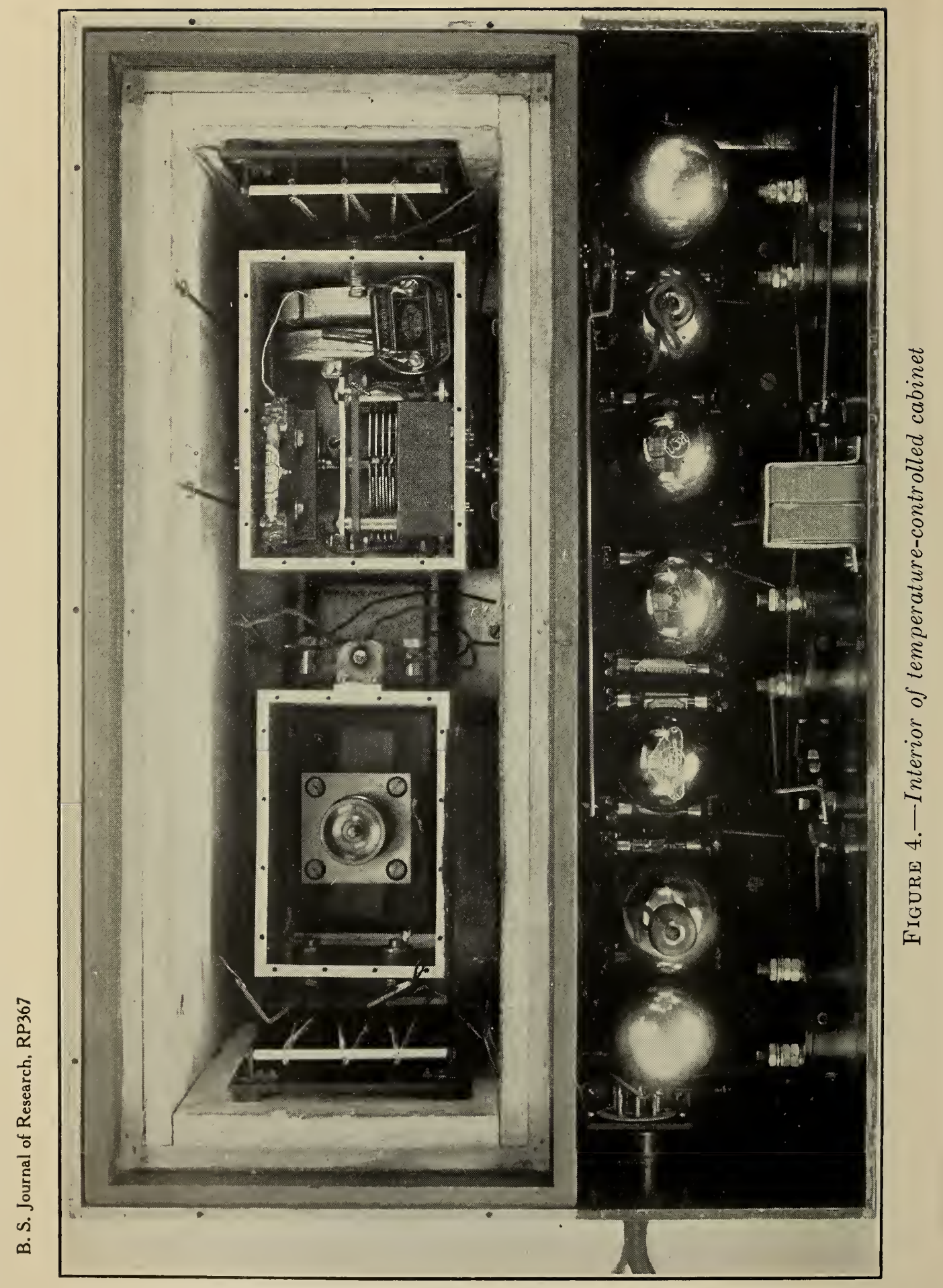


rectifier. This unit has been in continuous operation for four months, and in that time has not required any attention whatever.

The piezo electric element is a $30 \mathrm{kc}$ rectangular bar, $30^{\circ}$ or $\mathrm{Y}$ cut. ${ }^{3}$ It is mounted in a special mounting (fig. 7) in such a way that its

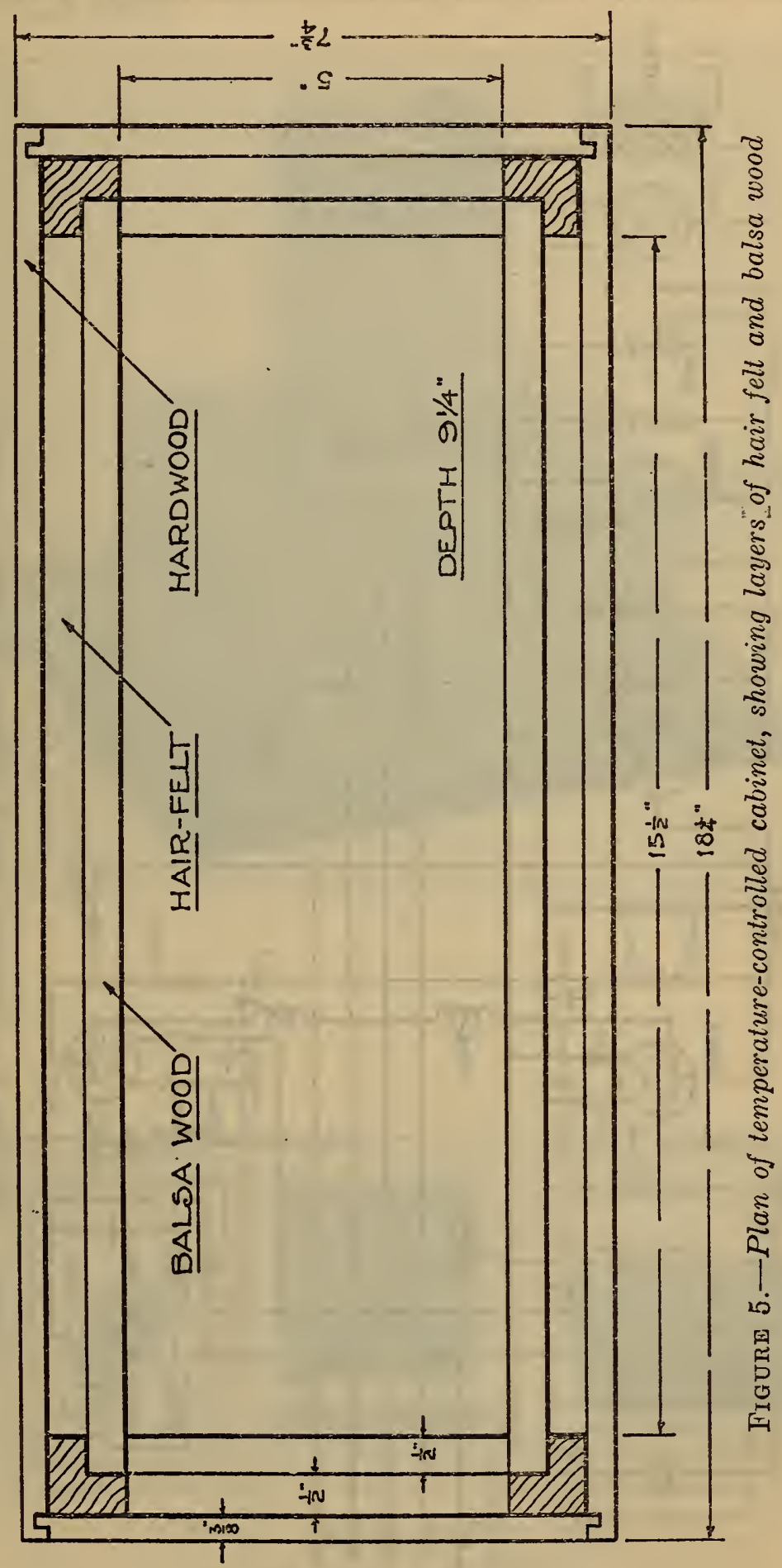

frequency is practically free from any changes due to jarring. A spring and key in the upper electrode presses the quartz plate against a narrow, raised surface on the lower electrode. As shown in the

W. G. Cady, Piezo-electric Terminology, Proc. I. R. E., vol. 18, p. 2136; December, 1930. 
figure, the central portion of the contacting areas are ground away so that pressure is applied only near the edges of the plate. The spring is a phosphor-bronze helix so designed that the pressure is as nearly independent of temperature as possible. In this way the quartz plate is held midway between the electrodes, and is quite free to vi-

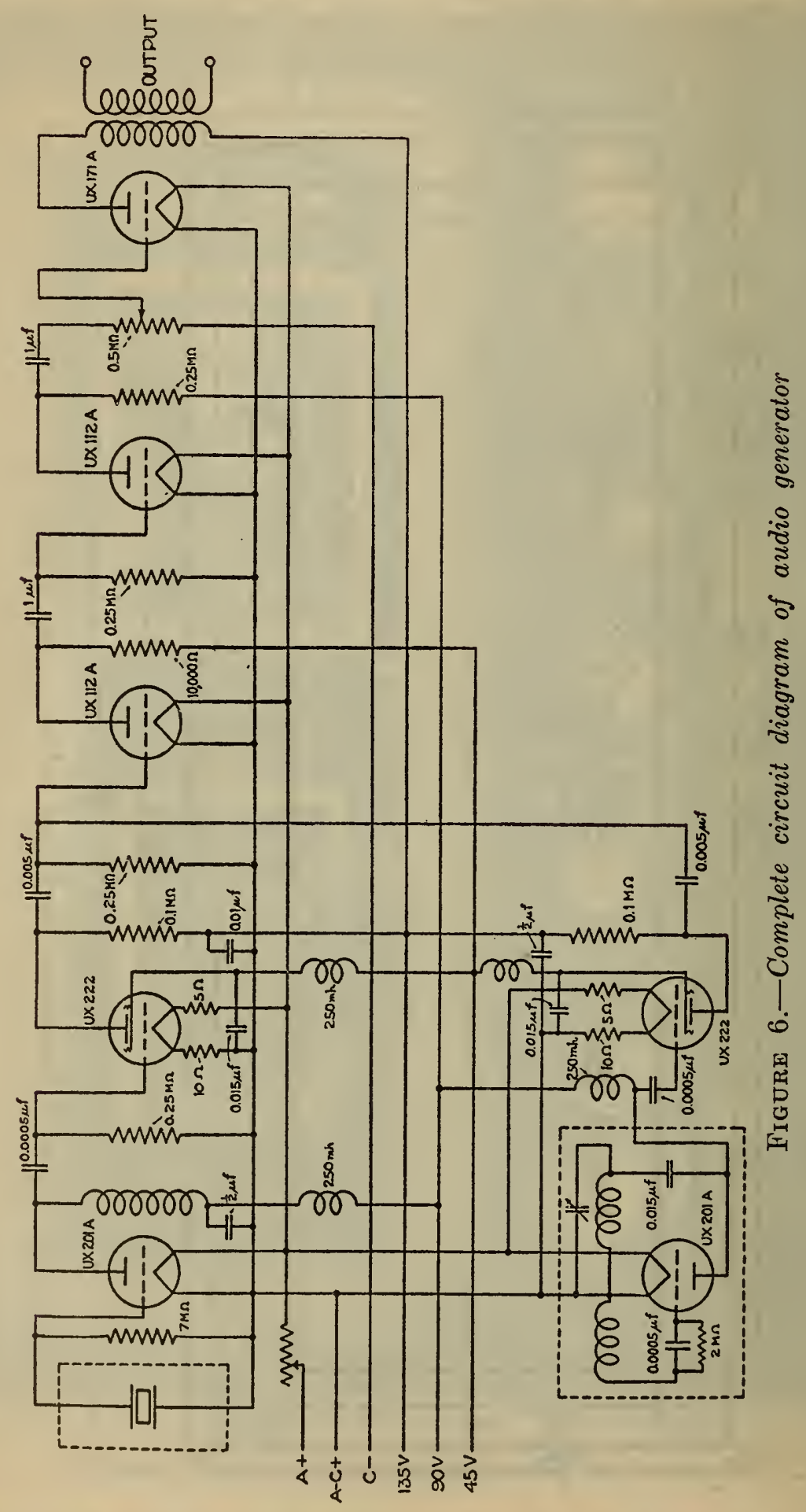

brate at its extensional mode. The electrodes are spaced with doughnut-shaped pyrex insulators. The piezo oscillator circuits (see fig. 6) are the same as those used in the secondary standards. ${ }^{4}$

IV. E. Heaton and W. H. Brattain, Design of a Portable Temperature-controlled Piezo Oscillator, Proc. I. R. E., vol. 18, p. 345; July, 1930. B. S. Jour. Research, vol. 4, (RP153); March, 1930. 

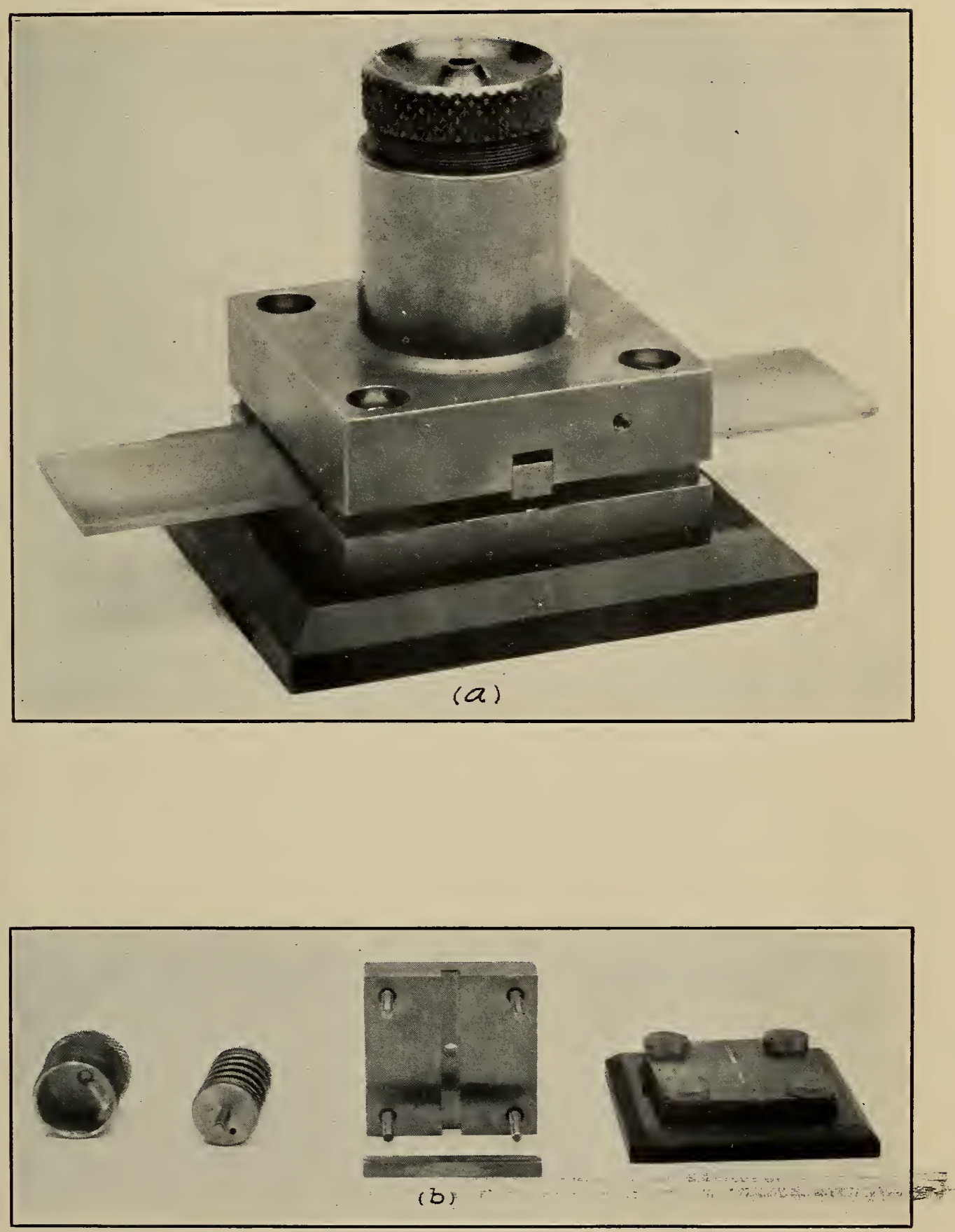

FIgURE 7.-Quartz plate mounting

$a$, Assembled; $b$, Disassembled. 
The variable oscillator is a modified Hartley circuit (fig. 6), the frequency of which can be varied from approximately 28.50 to 30.00 kc. The tuning capacity is a $250 \mu \mu \mathrm{f}$, straight-line frequency, receiving condenser connected in parallel with a $200 \mu \mu \mathrm{f}$ mica condenser. The hard rubber insulation which was used in the variable condenser has been replaced by pyrex insulation in order that the oscillator might maintain its calibration over a longer period of time. A $15 \mu \mu$ variable condenser is connected in parallel with the other tuning capacities in order that the audio output may be adjusted to zero cycles per second when the main tuning condenser is set on the zero position. If some other audio-frequency standard is available, the output can be adjusted to exact agreement with that point on the calibration curve instead. The adjustment for this condenser is a bakelite rod brought out through the back of the case. The range of the audiofrequency generator, which, in this case, is only from 50 to 1,500 cycles per second, can be extended as desired by adjusting the capacities of the fixed and variable condensers.

The outputs of the two $30-\mathrm{kc}$ oscillators are amplified separately and fed into a common detector. The resulting audio-frequency note is passed through a two-stage resistance-coupled amplifier. The resistance-capacity coupling is used in order to obtain approximately constant amplification over the entire audio-frequency range. A transformer is used in the output circuit to prevent any change in beat note due to a change in external connections.

The quality of the audio-frequency note which is obtained is largely dependent on the audio-frequency amplifier. If a pure sinusoidal wave-form were required it is probable that some refinements in the amplifier would have to be made. However, no attempt has been made to determine the exact wave-form under the present conditions. For the purpose for which the audio-frequency generator was designed, the quality of the note is entirely satisfactory.

The calibration of the audio-frequency generator has been checked repeatedly for some time. The original calibration and all recalibrations are made against harmonics of the 100-cycle-per-second output of the primary frequency standard. These measurements are made in the following manner. The condenser is set on the setting for 100 cycles per second, which is obtained from the original calibration curve. The audio-frequency note is then adjusted to exact agreement with the standard by means of the small auxiliary condenser previously described. The settings for harmonics of the 100 cycles are then determined. For any setting of the condenser the frequency has been found to vary less than 0.1 cycle per second. Furthermore, if the plate voltage is maintained at a constant value, the auxiliary condenser will not have to be readjusted for several hours at a time, which permits one to make measurements over an extended period without interruption.

Washington, July 8, 1931.

$74280-31-6$ 